\title{
INFLUENCIA DEL AMBIENTE DE TRABAJO SOBRE EL EMPODERAMIENTO Y SOBRE LA INFUSIÓN DE SISTEMAS DE INFORMACIÓN*
}

\author{
INFLUENCE OF WORK ENVIRONMENT ON EMPOWERMENT \\ AND ON INFORMATION SYSTEMS INFUSION
}

\author{
ALBERTO UN JAN \\ Universidad Norbert Wiener \\ GABRIELA CAUVI \\ Universidad Norbert Wiener
}

\section{RESUMEN}

Las empresas invierten altos montos de dinero en la implementación de sistemas y tecnologías de información, según Gartner ${ }^{3}$, pero no aprovechan todo su potencial porque los sistemas recientemente implementados no llegan al punto de infusión, conforme señalaron Kim y Gupta ${ }^{6}$. La infusión es el uso del sistema en su completo potencial, de manera extendida, integrativa y emergente.

Este artículo utiliza el instrumento propuesto por Kim y Gupta ${ }^{6}$ para medir la influencia del ambiente de trabajo sobre el empoderamiento, y la influencia del empoderamiento sobre la infusión de sistemas de información. Se obtiene así un mecanismo para lograr la exitosa infusión de los sistemas de información.

Los datos para validar las hipótesis fueron recolectados por medio de una encuesta a usuarios de diferentes sistemas de información. Todos los usuarios son estudiantes de la maestría en Gestión de la Universidad Nacional Mayor de San Marcos, semestres 2015-2 y 2016-1, que laboran en empresas de la ciudad de Lima.

En el modelo resultante, el ajuste percibido es la variable del ambiente de trabajo que inicia el empoderamiento; se debe trabajar sobre el diseño del sistema de información para lograr que este se ajuste y coincida con el trabajo del usuario.

Esta coincidencia logrará el empoderamiento, y el empoderamiento permitirá el uso integrativo y extendido del sistema de información. Esto hará posible el uso emergente del sistema de información, para completar su infusión. Se concluye que al mejorar el ajuste percibido se mejora la infusión.

Palabras clave: ambiente de trabajo, empoderamiento, infusión, sistema de información.

\footnotetext{
* Los autores agradecen encarecidamente a Juana Reaño y Jannet Guerrero por su valiosa participación en el desarrollo de la presente investigación.
} 


\section{ABSTRACT}

Companies invest large amounts of money in implementing information systems and technologies, Gartner ${ }^{3}$; however, they do not take advantage of all their potential because the systems just implemented, do not reach the infusion point, Kim and Gupta ${ }^{6}$. Infusion refers to the use of systems in their complete potential, in an extended, integrative and emergent way.

This article uses the instrument proposed by Kim and Gupta ${ }^{6}$ to measure the influence of work environment on empowerment, and the influence of empowerment on information systems infusion. In this way, a mechanism to achieve the successful information systems infusion is obtained.

Data to validate the hypothesis are collected through a survey among users of different information systems. All the users are students of the Master in Science in Management at Universidad Nacional Mayor de San Marcos, semesters 2015-2 and 2016-1, who work for companies in Lima.

In the resulting model, perceived fitness is the variable from work environment that starts empowerment; therefore, work must be done on the information system design to achieve its fitness and coincidence with the work of the user. This coincidence will achieve empowerment, and empowerment will achieve integrative and extended use of the information system. These two new forms of use will achieve the emergent use of the information system, to complete its infusion. In conclusion, improving perceived fitness will improve infusion.

Key words: work environment, empowerment, infusion, information system.

\section{INTRODUCCIÓN}

Las empresas invierten sumas considerables en la implementación de sistemas y tecnologías de información. Gartner ${ }^{3}$ presenta las siguientes cifras a nivel mundial entre los ańos 2015 y 2016:

- Los gastos en seguridad para IoT alcanzarán \$348 millones

- Se espera que las ganancias en semiconductores declinen $0,6 \%$

- Los envíos de computadores personales (PC) declinaron 9,6\% en el primer trimestre de 2016

- Se pronostica que los gastos en tecnología de información (TI) declinarían en $0,5 \%$ para 2016

- El mercado de manufactura de equipos semiconductores declinó $1 \%$ en 2015
- Las ganancias por semiconductores declinaron $2.3 \%$ en 2015

- La venta global de teléfonos inteligentes crecería $7 \%$ en 2016

- Las ganancias por servidores crecieron $8.2 \%$ en el cuarto trimestre de 2015, mientras que los envíos crecieron 9,2\%.

- La venta de teléfonos inteligentes creció 9,7\% en el cuarto trimestre de 2015.

- La inteligencia de negocios y el mercado analítico alcanzaría 16.9 mil millones de dólares en 2016.

- La venta de dispositivos portátiles crecería $18.4 \%$ en 2016

- El mercado de servicios públicos en la nube alcanzaría 204 mil millones de dólares para el 2016 
- Los envíos de dispositivos crecerían 1,9\% en 2016

- El gasto en TI crecería 0,6 \% en 2016

- Los envíos de PC declinaron 8,3 \% en el cuarto semestre de 2015

- El gasto de capital para semiconductores declinaría 4,7\% en 2016

- Los ingresos por semiconductores declinaron $1,9 \%$ en 2015

Kim y Gupta ${ }^{6}$ encontraron que las inversiones no aprovechan todo su potencial porque los sistemas recientemente implementados, luego de ser introducidos, no llegan al punto de infusión en la vida de trabajo de los empleados. Según el modelo de implementación de sistemas de información de Saga y Zmud', las empresas pueden aprovechar sus inversiones en sistemas de información solo en el punto de infusión de sistemas de información. La infusión se refiere al uso del sistema en su completo potencial, de manera extendida, integrativa y emergente. Kim y Gupta $^{6}$ señalan que una barrera para la infusión de sistemas de información es que los sistemas de premios en las empresas no reconocen los esfuerzos del individuo que intenta utilizar el sistema más allá del uso establecido, y los individuos que intentan hacerlo corren riesgos en términos de su evaluación en el trabajo o la pérdida de su propio tiempo que dedican a tal esfuerzo. Un mejor entendimiento de la infusión de sistemas de información requiere tomar en cuenta una orientación motivacional activa, la cual está representada por el concepto de empoderamiento psicológico. Kim y Gupta ${ }^{6}$ proponen el empoderamiento del usuario como una extensión conceptual del empoderamiento psicológico, al examinar la infusión de los sistemas de información. Como una interpretación de la infusión de sistemas de información, señalan que "el empoderamiento del usuario deberá inspirar a los usuarios a ir más allá del uso establecido de los sistemas de información para explotar el potencial completo del sistema en el trabajo".

Este artículo utiliza el instrumento de medición propuesto por Kim y Gupta (2014) para medir la influencia del ambiente de trabajo sobre el empoderamiento, y la influencia del empoderamiento sobre la infusión de sistemas de información. De esta manera se tiene un mecanismo para lograr la exitosa infusión de los sistemas de información.

La pregunta de investigación es la siguiente: ¿cuál es la influencia del ambiente de trabajo sobre el empoderamiento y sobre la infusión de sistemas de información?

El objetivo de la investigación será hallar la influencia del ambiente de trabajo sobre el empoderamiento y sobre la infusión de sistemas de información.

El diseño de un ambiente de trabajo afecta al desarrollo del empoderamiento del usuario. Este empoderamiento, a su vez, regula aspectos de la infusión de los sistemas de información. Kim y Gupta ${ }^{6}$ definen empoderamiento como una orientación motivacional activa hacia el uso de una aplicación de tecnología de información en el trabajo, y aclaran respecto a la naturaleza del empoderamiento del usuario y su impacto sobre la infusión de los sistemas de información. El modelo utilizado demuestra las hipótesis propuestas por Kim y Gupta ${ }^{6}$; estas hipótesis han sido adecuadas a la realidad de las empresas peruanas consideradas en este estudio. La sección II (Marco teórico)explica los conceptos de infusión y empoderamiento 
dentro de un ambiente de trabajo. La sección III (Modelo de investigación) muestra y explica la relación entre las variables del ambiente de trabajo y el empoderamiento, sugiriendo además las variables de control; se propone las hipótesis que se desea demostrar. La sección IV (Material y método) sugiere la muestra a utilizar y las pruebas estadísticas realizadas. La sección $\mathrm{V}$ (Resultados) analiza los resultados del tratamiento estadístico. La sección VI (Discusión) presenta la interpretación de resultados para confirmar las hipótesis que se demostraron y mostrar el modelo validado. Finalmente, las conclusiones se presentan en la sección VII.

\section{MARCO TEÓRICO}

A continuación se presentan algunas definiciones necesarias para el modelo que se desea demostrar.

\section{Definición de infusión}

Según Kim y Gupta ${ }^{6}$, infusión es la etapa final de un proceso de desarrollo de sistemas de información, y se refiere al uso del sistema de información en su máximo potencial de manera extendida, integrati- va y emergente. En un modelo de implementación de sistemas de información, el sistema (adoptado) será adaptado, aceptado, hecho rutina y finalmente ocurrirá la infusión por los usuarios. En esta última etapa, el individuo utiliza totalmente el sistema para enriquecer el rendimiento de las tareas. Entiéndase infusión como el uso del máximo potencial, que puede darse de tres maneras distintas: uso extendido, uso integrativo y uso emergente, según se describe en la Tabla 1.

Infusión representa una forma de comportamiento proactivo que significa "las acciones del individuo" que afectan los cambios ambientales por medio de la búsqueda de oportunidades, mostrando iniciativas, tomando acción y resolviendo problemas, y perseverando hasta lograr el cambio. La definición anterior se limita a la tecnología de los sistemas de información. Otros autores amplían la definición de una manera más general, utilizando conceptos de tecnología e innovación. Así, por ejemplo, Jones, Sundaram y Chin ${ }^{4}$ amplían el concepto de infusión a la innovación: "Infusión es la extensión con la cual el potencial completo de una innovación se ha incluido en el sistema de trabajo operacional o gerencial de una organización"; y para medir

TABLA 1

Formas de usar un sistema en todo su potencial para lograr la infusión, más allá del uso estándar o rutinario. Mencionado por Kim y Gupta (2014) quienes citan a Saga y Zmud (1990)

\begin{tabular}{c|c|c} 
Uso extendido & Uso integrativo & Uso emergente \\
\hline $\begin{array}{c}\text { Usar más de las caracte- } \\
\text { rísticas disponibles del } \\
\text { sistema para completar las } \\
\text { tareas }\end{array}$ & $\begin{array}{c}\text { Uso del sistema para reforzar } \\
\text { enlaces entre tareas }\end{array}$ & $\begin{array}{c}\text { Uso de un sistema de } \\
\text { manera nueva e innovadora } \\
\text { para apoyar a las tareas }\end{array}$ \\
& &
\end{tabular}


el constructo "infusión del nuevo sistema" utilizan el siguiente instrumento de cuatro afirmaciones con escala de Likert de siete valores, aplicado al concepto tecnología:

1. Estoy usando [la tecnología] en su máximo potencial para apoyar mi propio trabajo.

2. Estoy usando todas las capacidades [de la tecnología] de la mejor manera para ayudarme en el trabajo.

3. Dudo de que haya mejores maneras de usar [la tecnología] para apoyar mi trabajo.

4. Mi uso de [la tecnología] en el trabajo ha sido integrado e incorporado al más alto nivel.

\section{Definición de empoderamiento}

Alsop y Heinsohn ${ }^{1}$, en un documento del Banco Mundial, definen empoderamiento como "enriquecimiento de la capacidad del grupo o individuo o grupo para hacer elecciones y transformarlas en acciones deseadas y resultados". Además, el empoderamiento de los empleados contribuye al éxito organizacional; existe una relación directa entre el nivel de empoderamiento del empleado y el rendimiento del empleado, la satisfacción del empleado y el compromiso del empleado, según afirmaron Meyerson y Dewettinck ${ }^{8}$, quienes además señalaron que el empoderamiento es crítico para la innovación y efectividad organizacional.

El artículo de Kim y Gupta ${ }^{6}$ refuerza el concepto de innovación y efectividad, al definir empoderamiento como "orientación motivacional activa hacia el uso de una aplicación de tecnología de información en el trabajo".

Empoderamiento se muestra como tácticas de administración, tal como delegación, compartir información y participación de los empleados. El empoderamiento psicológico del usuario se manifiesta por medio de cogniciones que reflejan la

TABLA 2

Manifestaciones del empoderamiento psicológico a través de cogniciones que reflejan la orientación del individuo hacia su trabajo

\begin{tabular}{c|c} 
Cognición & Definición \\
\hline $\begin{array}{c}\text { Competencia del usuario } \\
\text { (CMP) }\end{array}$ & $\begin{array}{c}\text { Creencia del individuo en su capacidad } \\
\text { para usar el sistema en tareas con }\end{array}$ \\
\hline $\begin{array}{c}\text { Impacto en el usuario del sistema } \\
\text { (IMP) }\end{array}$ & $\begin{array}{c}\text { Grado con el cual un individuo puede } \\
\text { influenciar los resultados de una tarea } \\
\text { basado en el uso del sistema }\end{array}$ \\
\hline $\begin{array}{c}\text { Significado del uso del sistema } \\
\text { (MNG) }\end{array}$ & $\begin{array}{c}\text { Importancia que un individuo le asigna } \\
\text { al uso de un sistema en relación }\end{array}$ \\
\hline $\begin{array}{c}\text { Autodeterminación del usuario } \\
\text { (SDT) }\end{array}$ & a sus propios ideales o estándares \\
\hline
\end{tabular}


orientación del individuo hacia su trabajo, conforme señalaron Kim y Gupta ${ }^{6}$, Lau y Lew $^{7}$, Dude ${ }^{2}$, Ke y Zhang ${ }^{5}$, y Spreitzer ${ }^{10}$. Estas cogniciones se identifican y definen en la Tabla 2.

\section{Ambiente de trabajo}

El ambiente de trabajo se clasifica en tres aspectos de diseño: 1) Diseño del trabajo; 2) diseño de la estructura social organiza-

TABLA 3

Clasificación del ambiente de trabajo según el aspecto

\begin{tabular}{l|l|l}
\multicolumn{1}{c|}{ Aspecto } & $\begin{array}{l}\text { Factores representativos } \\
\text { de cada aspecto }\end{array}$ & \multicolumn{1}{c}{ Definición del factor representativo } \\
\hline 1. Diseño del trabajo & Autonomía en el trabajo & $\begin{array}{l}\text { Grado con el que el trabajo proporciona li- } \\
\text { bertad substancial, independencia y discre- } \\
\text { ción al individuo en la elaboración de ca- } \\
\text { lendarios de trabajo y en la determinación } \\
\text { de procedimientos a ser utilizados para } \\
\text { hacer dicho trabajo. }\end{array}$ \\
\hline $\begin{array}{l}\text { 2. Diseño de la estructura } \\
\text { social organizacional }\end{array}$ & Clima para aportar & $\begin{array}{l}\text { Grado con el que los miembros de las or- } \\
\text { ganizaciones son vistos en su intento de } \\
\text { destacar, enfrentar problemas difíciles, y } \\
\text { progresar. }\end{array}$ \\
\hline $\begin{array}{l}\text { 3. Diseño del sistema de } \\
\text { información }\end{array}$ & Ajuste percibido & $\begin{array}{l}\text { Grado con que el sistema coincide con el } \\
\text { trabajo del usuario. }\end{array}$
\end{tabular}

cional; y 3) diseño del sistema de información. Cada aspecto de diseño posee un factor representativo. El modelo de investigación describe el ambiente de trabajo con los factores representativos de cada aspecto que se mencionan en la Tabla 3.

La autonomía en el trabajo se refiere a la discreción con la que se puede elegir métodos y procedimientos para completar un trabajo; se puede elegir el horario y la secuencia de actividades de trabajo, y se puede elegir el criterio con el que se evalúa el trabajo ${ }^{2}$.

\section{MODELO DE INVESTIGACIÓN}

Con las definiciones anteriores surge el modelo de la Figura 1.

\section{Antecedentes de empoderamiento de usuario}

Kim y Gupta ${ }^{6}$ identifican las siguientes relaciones entre los aspectos del ambiente del trabajo y el empoderamiento, expresadas como hipótesis:

H1: El ajuste percibido tiene un efecto positivo sobre el empoderamiento del usuario.

H2: La autonomía en el trabajo tiene un efecto positivo sobre el empoderamiento del usuario.

H3: El clima para aportar tiene un efecto positivo sobre el empoderamiento del usuario. 
FIGURA 1

Modelo de investigación de Kim y Gupta (2014)

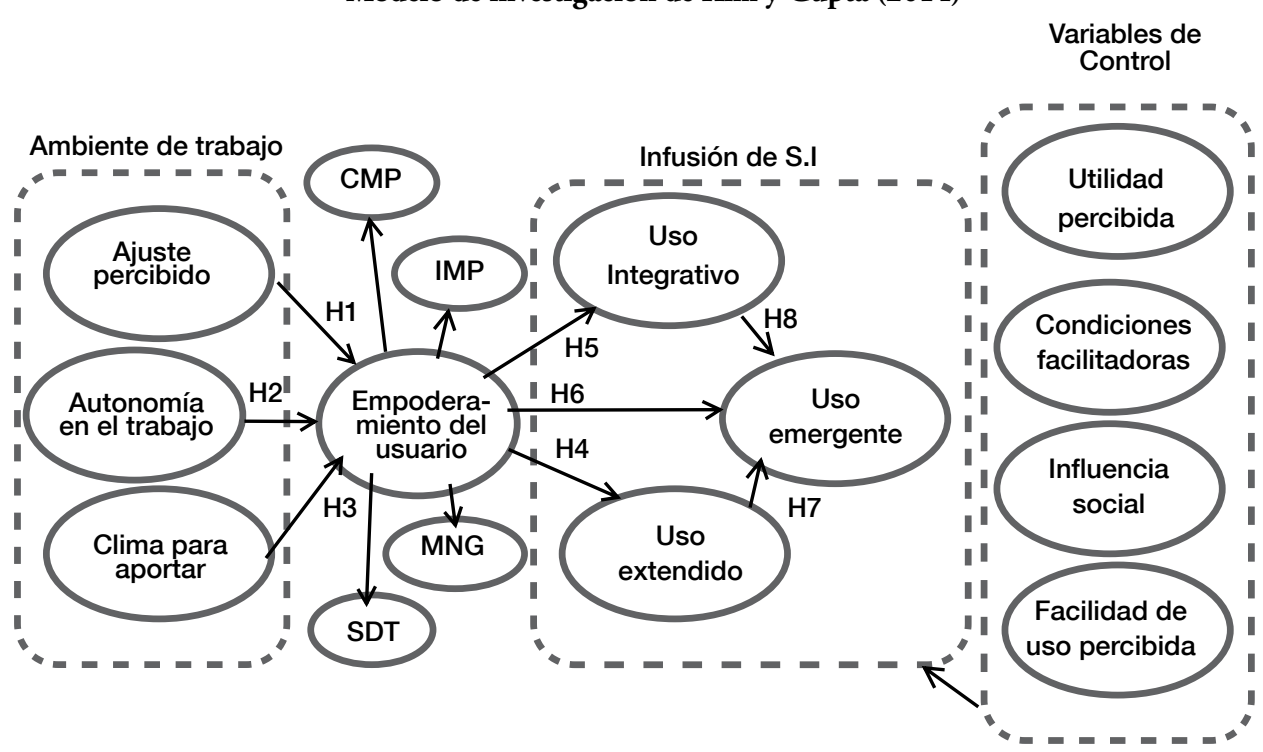

SI: Sistema de información. CMP: Competencia del usuario. IMP: Impacto en el uso del sistema. MNG: Significado del uso del sistema. SDT: Autodeterminación del usuario.

\section{Consecuencias del Empoderamiento de los usuarios}

Como consecuencia del empoderamiento se obtienen las siguientes hipótesis:

H4: El empoderamiento de los usuarios tiene un efecto positivo sobre el uso extendido.

H5: El empoderamiento de los usuarios tiene un efecto positivo sobre el uso integrativo.

H6: El empoderamiento de los usuarios tiene un efecto positivo sobre el uso emergente.

H7: El uso extendido tiene un efecto positivo sobre el uso emergente.

H8: El uso integrativo tiene un efecto positivo sobre el uso emergente.

\section{Control}

El modelo original añade cuatro constructos como variables de control: utilidad percibida, facilidad de uso percibida, condiciones facilitadoras, e influencia social. También propone los instrumentos de medida para estas cuatro variables. Si bien no hay una hipótesis sobre la relación de las variables de control con las variables de empoderamiento, Kim y Gupta ${ }^{6}$ encuentran que la facilidad de uso percibida tiene efecto significativo sobre el uso extendido: correlación 0.54 ; y la utilidad percibida tiene efecto significativo sobre el uso integrativo: correlación 0.62 .

\section{MATERIAL Y MÉTODO}

Los datos para validar empíricamente las hipótesis son recolectados por medio de 
TABLA 4 (parte 1 de 4)

Encuesta: Ambiente de trabajo, empoderamiento e infusión (Se usó escala de Likert del 1 al 5.

Adaptada para su publicación)

Estimado participante: Por favor, llene sus datos y los de la institución para la que trabaja.

\begin{tabular}{l|l|l|l}
\hline Rango de edad del participante & & Institución para la que trabaja & \\
\hline Género & & Su actual cargo en la institución & \\
\hline Profesión & $\begin{array}{l}\text { Tiempo (años) que trabaja en la } \\
\text { institución }\end{array}$ & \\
\hline
\end{tabular}

En esta encuesta se le preguntará acerca de su interacción con un sistema de información.

¿Cuál es el sistema de información que desea describir en esta encuesta?

\section{ADECUACIÓN PERCIBIDA DEL SISTEMA DE INFORMACIÓN}

EI SISTEMA DE INFORMACIÓN se adecúa a los requerimientos de mi trabajo

EI SISTEMA DE INFORMACIÓN es compatible con mi trabajo

En general, el SISTEMA DE INFORMACIÓN se adecúa a mi trabajo

\section{AUTONOMÍA EN EL TRABAJO}

Mi trabajo me da la oportunidad de usar mi iniciativa y juicio personal para la realización de mis tareas

Tengo mucha autonomía en mi trabajo

Mi trabajo me da considerable oportunidad de tener mi independencia y libertad para hacer mis tareas

\section{CLIMA PARA EL LOGRO}

Las personas en esta organización intentan ser excelentes en el trabajo

Las personas en esta organización intentan enfrentar problemas difíciles

Las personas en esta organización intentan progresar

\section{COMPETENCIA DE LOS USUARIOS EN EL USO DEL SISTEMA DE INFORMACIÓN}

He dominado las habilidades necesarias para usar el SISTEMA DE INFORMACIÓN

Estoy muy seguro de mis capacidades para usar el SISTEMA DE INFORMACIÓN

Tengo confianza en mi habilidad para usar el SISTEMA DE INFORMACIÓN 
TABLA 4 (parte 2 de 4 )

Encuesta: Ambiente de trabajo, empoderamiento e infusión

(Se usó escala de Likert del 1 al 5.

Adaptada para su publicación)

\section{IMPACTO EN EL USO DEL SISTEMA DE INFORMACIÓN}

Usando el SISTEMA DE INFORMACIÓN, mi impacto sobre lo que sucede en el trabajo es grande Usando el SISTEMA DE INFORMACIÓN, tengo significativa influencia sobre lo que sucede en el trabajo

Usando el SISTEMA DE INFORMACIÓN, tengo un alto nivel de control sobre lo que sucede en el trabajo

\section{SIGNIFICADO DEL USO DEL SISTEMA DE INFORMACIÓN}

EI SISTEMA DE INFORMACIÓN que utilizo es muy importante para mí

EI SISTEMA DE INFORMACIÓN que utilizo tiene significado para mí

Mis actividades en el SISTEMA DE INFORMACIÓN tienen mucho significado personal para mí

\section{AUTODETERMINACIÓN}

Tengo significativa autonomía al determinar cómo utilizo el SISTEMA DE INFORMACIÓN en el trabajo

Tengo oportunidad de independencia y libertad en la forma de usar el SISTEMA DE INFORMACIÓN para el trabajo

Puedo decidir por mi cuenta cómo utilizar el SISTEMA DE INFORMACIÓN en mi trabajo

\section{USO EXTENDIDO DEL SISTEMA DE INFORMACIÓN}

Utilizo la mayor cantidad de características disponibles en el SISTEMA DE INFORMACIÓN para apoyar mis tareas

Utilizo todas las características disponibles del SISTEMA DE INFORMACIÓN para ayudarme en la ejecución de mis tareas

Utilizo intensamente las características disponibles en el SISTEMA DE INFORMACIÓN para completar mis tareas 
TABLA 4 (parte 3 de 4)

Encuesta: Ambiente de trabajo, empoderamiento e infusión

(Se usó escala de Likert del 1 al 5.

Adaptada para su publicación)

\section{USO INTEGRADOR DEL SISTEMA DE INFORMACIÓN}

Utilizo el SISTEMA DE INFORMACIÓN para tener mejores conexiones entre tareas

Utilizo el SISTEMA DE INFORMACIÓN para unir tareas relacionadas

Utilizo el SISTEMA DE INFORMACIÓN para coordinar tareas múltiples

\section{USO EMERGENTE DEL SISTEMA DE INFORMACIÓN}

Exploro cómo el SISTEMA DE INFORMACIÓN puede apoyar mejor mi trabajo

Con frecuencia encuentro nuevos usos del SISTEMA DE INFORMACIÓN para apoyar mi trabajo

Utilizo el SISTEMA DE INFORMACIÓN de manera novedosa para realizar mis tareas

\section{CONDICIONES QUE FACILITAN EL USO DEL SISTEMA DE INFORMACIÓN}

Una persona o grupo específico está disponible para asistencia con las dificultades del SISTEMA DE INFORMACIÓN

Tengo guías disponibles para el uso del SISTEMA DE INFORMACIÓN

Hay instrucciones especializadas en relación al SISTEMA DE INFORMACIÓN disponibles para mí

\section{UTILIDAD PERCIBIDA DEL SISTEMA DE INFORMACIÓN}

Encuentro el SISTEMA DE INFORMACIÓN útil en mi trabajo

El uso del SISTEMA DE INFORMACIÓN me permite completar tareas más rápidamente

Usar el SISTEMA DE INFORMACIÓN aumenta mi productividad

TABLA 4 (parte 4 de 4)

Encuesta: Ambiente de trabajo, empoderamiento e infusión

(Se usó escala de Likert del 1 al 5.

Adaptada para su publicación)

\section{INFLUENCIA SOCIAL}

Personas que influencian mi comportamiento piensan que debería usar el SISTEMA DE INFORMACIÓN

Personas importantes para mí piensan que yo debería usar el SISTEMA DE INFORMACIÓN

La gerencia general de este negocio ha apoyado en el uso del SISTEMA DE INFORMACIÓN 
una encuesta a usuarios de diferentes sistemas de información. La Tabla 4 muestra los constructos de la encuesta que se repartió.

Todos los usuarios son estudiantes de la maestría en Gestión de la Universidad Nacional Mayor de San Marcos, semestres 2015-2 y 2016-1. Los usuarios laboran en empresas de la ciudad de Lima. Se determinó el tamaño de la muestra con la fórmula (1):

$$
\mathrm{n}=\mathrm{z}^{2} \sigma^{2} / \mathrm{d}^{2}
$$

Con $\mathrm{z}=1.65$ para un coeficiente de confianza $0.90, \sigma=3$ puntos en la encuesta, $\mathrm{y} d=1$ punto en la encuesta, se obtiene un tamaño de muestra $\mathrm{n}=24.5$.

Se recibieron 31 respuestas. La profesión de los encuestados y el tiempo de trabajo en la empresa se muestran en las Tablas 5 y 6.

Luego de recolectar los datos, se aplicó el alfa de Cronbach a los grupos de datos; cada variable propuesta tiene un grupo de tres preguntas. La encuesta midió 14 constructos, todos con un alfa de Cronbach

\section{TABLA 5}

Profesión del encuestado

\begin{tabular}{c|c} 
Profesión & Frecuencia \\
\hline Administración & 11 \\
\hline $\begin{array}{c}\text { Bibliotecología / } \\
\text { Comunicaciones }\end{array}$ & 4 \\
\hline $\begin{array}{c}\text { Economía / Conta- } \\
\text { bilidad }\end{array}$ & 2 \\
\hline Ingeniería & 3 \\
\hline Tecnología médica & 2 \\
\hline Docente & 31
\end{tabular}

mayor a 0.7 Para medir la relación de las variables de dos en dos se aplicó una matriz de correlación.

\section{RESULTADOS}

Con los promedios de los grupos de datos se elaboró una matriz de correlación, la cual muestra las relaciones que existen entre las variables del modelo, y que son las relaciones cuya existencia queremos demostrar. Se obtiene la matriz de correlación de la Tabla 7.

\section{DISCUSIÓN}

La matriz de correlación confirmó las hipótesis $\mathrm{H} 1, \mathrm{H} 4, \mathrm{H} 5, \mathrm{H} 7$ y H8. No se confirmaron las hipótesis H2, H3, H6.

En el modelo resultante se observa que el ambiente de trabajo se describe con la variable ajuste percibido, lo cual confirma la hipótesis H1. En el ambiente de trabajo no interviene la autonomía en el trabajo ni el clima para aportar, por lo que se descartan las hipótesis $\mathrm{H} 2$ y H3.

\section{TABLA 6}

Tiempo en la empresa

\begin{tabular}{c|c} 
Tiempo (años) & Frecuencia \\
\hline $0,1 \circ 2$ años & 11 \\
\hline 3 o 4 años & 10 \\
\hline $5 \circ 6$ años & 2 \\
\hline 7 u 8 años & 1 \\
\hline 9 o 10 años & 4 \\
\hline 11 o 12 años & 1 \\
\hline No responde & 2
\end{tabular}


TABLA 7

Matriz de correlación

\begin{tabular}{|c|c|c|c|c|c|c|c|c|c|c|c|c|c|c|}
\hline & FIT & JAU & CLM & СMP & IMP & MNG & SDT & EXT & ITG & EMG & FCD & USF & EOU & SIF \\
\hline FIT & 1 & \begin{tabular}{|l|}
-.04 \\
\end{tabular} & .05 & .27 & .69 & .50 & .37 & .21 & .35 & \begin{tabular}{|l|}
.08 \\
\end{tabular} & .63 & .76 & .21 & .30 \\
\hline JAU & & 1 & .14 & \begin{tabular}{|l|}
.02 \\
\end{tabular} & .14 & .02 & .42 & .14 & -.20 & .08 & .12 & -.13 & -.16 & .19 \\
\hline CLM & & & 1 &.- .13 & \begin{tabular}{|l}
.04 \\
\end{tabular} & .11 & -.09 & -.10 & .18 & -.18 & \begin{tabular}{|l|}
35 \\
\end{tabular} & .13 & \begin{tabular}{|l|}
.07 \\
\end{tabular} & .40 \\
\hline CMP & & & & 1 & .28 & -.05 & .26 & \begin{tabular}{|l|}
.05 \\
\end{tabular} & .17 & .33 & \begin{tabular}{|l|}
.09 \\
\end{tabular} & .35 & .44 & .31 \\
\hline IMP & & & & & 1 & .66 & .45 & .47 & .48 & .28 & .48 & .73 & .29 & .45 \\
\hline MNG & & & & & & 1 & .36 & .60 & .57 & .46 & .50 & \begin{tabular}{|l|}
.65 \\
\end{tabular} & .23 & .52 \\
\hline SDT & & & & & & & 1 & .42 & .18 & .42 & .43 & .46 & \begin{tabular}{|l|}
.02 \\
\end{tabular} & .40 \\
\hline EXT & & & & & & & & 1 & .56 & \begin{tabular}{|c|}
.69 \\
\end{tabular} & \begin{tabular}{|l|}
.37 \\
\end{tabular} & .41 & .28 & .42 \\
\hline ITG & & & & & & & & & 1 & .52 & .31 & \begin{tabular}{|l}
.64 \\
\end{tabular} & .50 & .57 \\
\hline EMG & & & & & & & & & & 1 & .21 & .33 & .40 & .51 \\
\hline FCD & & & & & & & & & & & 1 & .65 & -.10 & .57 \\
\hline USF & & & & & & & & & & & & 1 & .43 & .60 \\
\hline EOU & & & & & & & & & & & & & 1 & .33 \\
\hline SIF & & & & & & & & & & & & & & 1 \\
\hline$\alpha \mathrm{Cr}$ & .932 & \begin{tabular}{|l|}
.841 \\
\end{tabular} & .877 & .774 & \begin{tabular}{|l|l}
.890 \\
\end{tabular} & .829 & .913 & .878 & .845 & .886 & .838 & .936 & .715 & .700 \\
\hline
\end{tabular}

\begin{tabular}{c|c} 
FIT & Ambiente de trabajo: Ajuste percibido \\
\hline JAU & $\begin{array}{c}\text { Ambiente de trabajo: Autonomía en el } \\
\text { trabajo }\end{array}$ \\
\hline CLM & Ambiente de trabajo: Clima para aportar \\
\hline CMP & $\begin{array}{c}\text { Empoderamiento: Competencia del } \\
\text { usuario }\end{array}$ \\
\hline IMP & $\begin{array}{c}\text { Empoderamiento: Impacto en el uso del } \\
\text { sistema }\end{array}$ \\
\hline MNG & $\begin{array}{c}\text { Empoderamiento: Significado del uso } \\
\text { del sistema }\end{array}$ \\
\hline SDT & $\begin{array}{c}\text { Empoderamiento: Autodeterminación } \\
\text { del usuario }\end{array}$
\end{tabular}

Se observa también que en el empoderamiento, las variables competencia del usuario y autodeterminación del usuario no correlacionan con ninguna otra variable, por lo que su presencia no es necesaria en el modelo. Se confirma la influencia del empoderamiento del usuario sobre el uso integrativo y el uso extendido: se confirma H5 y confirma H4. Pero el empoderamiento no influye sobre el uso emergente, con lo que se descarta H6. Sin embargo, el uso emergente se origina, según $\mathrm{H} 7$ y $\mathrm{H} 8$, por la influencia del uso extendido y el uso integrativo. En las variables de control, las

\begin{tabular}{|c|c|}
\hline EXT & Infusión: Uso extendido \\
\hline ITG & Infusión: Uso integrativo \\
\hline EMG & Infusión: Uso emergente \\
\hline FCD & $\begin{array}{c}\text { Variable de control: Condiciones facili- } \\
\text { tadoras }\end{array}$ \\
\hline USF & Variable de control: Utilidad percibida \\
\hline EOU & Variable de control: Facilidad de uso \\
\hline SIF & Variable de control: Influencia social \\
\hline
\end{tabular}

condiciones facilitadoras tienen baja correlación con otras variables, y se pueden retirar del modelo.

Observando la Tabla 3, Clasificación del ambiente de trabajo según el aspecto, el ajuste percibido es un factor representativo del diseño del sistema de información, y se define como el grado con que el sistema coincide con el trabajo del usuario. En el modelo resultante, el ajuste percibido es la variable del ambiente de trabajo que inicia el empoderamiento; por tanto, se debe trabajar sobre el diseño del sistema de infor- 
FIGURA 2

Modelo resultante

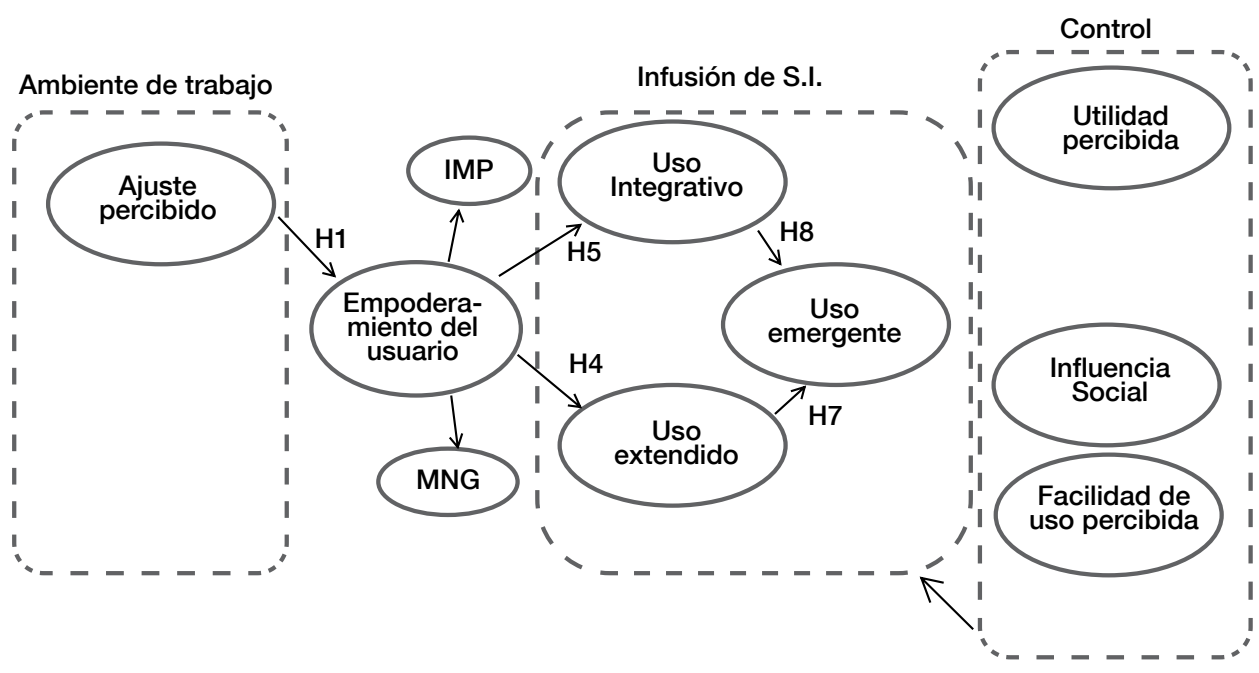

mación para lograr que coincida con el trabajo del usuario. Esta coincidencia logrará el empoderamiento, y el empoderamiento a su vez logrará el uso integrativo y el uso extendido del sistema de información. Estas dos últimas formas de uso lograrán el uso emergente del sistema de información, para completar la infusión del sistema de información. Se muestra el modelo resultante en la Figura 2.

\section{CONCLUSIONES}

La infusión se refiere al uso del sistema de información en su máximo potencial, de manera extendida, integrativa y emergente. En el modelo resultante, la variable ajuste percibido, que pertenece al ambiente de trabajo, tiene relación directa con el empoderamiento (con las variables impacto en el uso del sistema y significado del uso del sistema). El empoderamiento tiene relación directa con la infusión (con las va- riables uso integrativo y uso extendido). Las variables uso integrativo y uso extendido afectan al uso emergente. En conclusión, la variable que ingresa al modelo es el ajuste percibido, la cual, al incrementarse, mejorará la infusión de los sistemas de información. La infusión (según definición de la sección II) logrará el uso del sistema de información en su máximo potencial.

\section{REFERENCIAS BIBLIOGRÁFICAS}

1. Alsop R, Heinsohn N. Measuring Empowerment in Practice: Structuring Analysis and Framing Indicators. World Bank Policy Research Working Paper 3510, February 2005.

2. Dude D. Organizational commitment of principals: The effects of job autonomy, empowerment, and distributive justice. Doctor of Philosophy thesis, University of Iowa, 2012. http://ir.uiowa.edu/ etd/2863. 
3. Gartner. "Gartner newsroom". Consulta: 14 de julio de 2016. Disponible en: <http:// www.gartner.com/newsroom/>.

4. Jones E, Sundaram S, Chin W. Factors leading to sales force automation use: A longitudinal analysis. Journal of personal selling \& sales management, vol. XXII, 2002, no. 3, pp. 145-156.

5. Ke W, Zhang P. Effects of Empowerment on Performance in Open-Source Software Projects. IEEE Transactions on Engineering Management. Vol. 58, No. 2, May 2011, p. 334-346.

6. Kim H, Gupta S. A User Empowerment Approach to Information Systems Infusion. IEEE Transactions on Engineering Management, vol. 61, no. 4, November 2014.

7. Lau E, Lew V. Empowerment and Professional Ethics in Functional Context of
Design, Cost, and Construction Working Teams. International Journal of Sociology Study, Vol. 1, No. 1, March 2013, p. 1-16.

8. Meyerson G, Dewettinck B. Effect of Empowerment on Employees Performance. Advanced Research in Economic and Management Sciences (REMS). Vol. 2. July 2012.

9. Saga V, Zmud R. The nature and determinants of IT acceptance, routinization and infusión. Diffusion, Trnsfer and Implementation of Information Technology. L. Levine, Ed. Amsterdam, The Netherlands: Elsevier, 1994, pp.67-86.

10. Spreitzer G. Psychological empowerment in the workplace: Dimensions, measurement, and validation. Academy of Management Journal, 1995. 38(5), 14421465. Doi: $10.2307 / 256865$. 